\title{
Effects of higher order Jahn-Teller coupling on the nuclear dynamics
}

\author{
Alexandra Viel ${ }^{\mathrm{a})}$ and Wolfgang Eisfeld ${ }^{\mathrm{b})}$ \\ Lehrstuhl für Theoretische Chemie, Technische Universität München, D-85747 Garching, Germany
}

(Received 31 October 2003; accepted 15 December 2003)

\begin{abstract}
In this paper effects of higher order Jahn-Teller coupling terms on the nonadiabatic dynamics are studied. Of particular interest is the case when the potential energy surfaces of the degenerate state show pronounced anharmonicity. In order to demonstrate the effects a two-dimensional $E \otimes e$ Jahn-Teller model system is treated which is based on the $e^{\prime}$ stretching vibration of the photoactive ${ }^{2} E^{\prime}$ state of $\mathrm{NO}_{3}$ as a realistic example. The sixth order $E \otimes e$ Jahn-Teller Hamiltonian is derived in the diabatic representation which is valid for any system with a $C_{3}$ rotation axis. This diabatization scheme is compared to lower-order Jahn-Teller Hamiltonians and to symmetry adapted as well as ad hoc approximations. Lower-order potentials result in pronounced quantitative and qualitative differences in the dynamics, including differences in the evolution of mean values, the autocorrelation functions (and thus the corresponding spectra), and the electronic population evolution. In the particular example treated, the results of fourth and fifth order potentials are very similar to the sixth order reference system. In contrast, the approximate sixth order Hamiltonians, though the corresponding adiabatic surfaces seem to be nearly identical, results in pronounced differences. The possible consequences for the dynamics of realistic systems with higher dimensionality are briefly discussed. (C) 2004 American Institute of Physics.
\end{abstract}

[DOI: $10.1063 / 1.1646371]$

\section{INTRODUCTION}

Nonadiabatic dynamics plays an important role in the physics and chemistry of excited states. While the electronic ground state of most chemical systems is energetically well separated from higher electronic states (at least in the equilibrium region), this is often not the case for excited states. One of the consequences can be the breakdown of the BornOppenheimer (BO) approximation for which conical intersections are a prominent example. A particularly simple case are degenerate electronic states which are due to the high symmetry of the molecular system.

Whenever electronic states are energetically well separated nuclear and electronic motions are essentially uncoupled and the BO approximation is valid. In this case the nuclear dynamics is mainly determined by a single potential energy surface (PES). However, in regions where the energetic separation of electronic states becomes small the dynamical or nonadiabatic coupling between them, induced by the nuclear motion, can become extremely strong and can no longer be ignored. In this situation the curvature of the adiabatic PES's becomes strongly dependent on the nuclear coordinates and even cusps (more precisely discontinuities of partial derivatives) are observed at the conical intersection. In such a region the nuclear dynamics is determined by more than one PES and the coupling strength controls the electronic population transfer between different states.

The cusps in the adiabatic representation and the strong

\footnotetext{
${ }^{a)}$ Current address: LPQ-IRSAMC, University P. Sabatier, 118 Route de Narbonne, F-31062 Toulouse, France.

Electronic mail: alexandra.viel@irsamc.ups-tlse.fr

b)Electronic mail: wolfgang.eisfeld@ch.tum.de
}

coupling between surfaces are rather unfavorable for the practical treatment of the nuclear dynamics. One possible solution is to construct diabatic states which have a smoother behavior with respect to nuclear distortions and remove the discontinuities in the derivatives. However, in general it is not possible to uniquely define strictly diabatic states ${ }^{1,2}$ and thus a number of different approaches were suggested in the literature. ${ }^{3}$ The coupling between adiabatic states can be directly computed by $a b$ initio calculations. ${ }^{4,5}$ However, this is computationally very demanding and is only suitable for very small systems which do not pose any problems in the solution of the electronic structure. Another approach is to use some property of the wave functions (dipole moments, ${ }^{6}$ transition dipole moments, ${ }^{7}$ CI coefficients ${ }^{8}$ ) for a small number of adiabatic states and construct diabatic states by imposing the condition that the chosen property has to vary smoothly over the entire range of the coordinate space. A critical review of such methods was given by Dobbyn and Knowles. ${ }^{9}$ Several further diabatization schemes in this spirit were suggested in the literature and a brief overview can be found in a recent paper of Thiel and Köppel. ${ }^{10}$ A more extensive review was published by Pacher, Cederbaum, and Köppel. ${ }^{3}$

The method used in the present paper can be called "diabatization by ansatz" and does not require properties of the electronic wave function. The diabatic potential energy matrix is derived by expanding the state energies in a Taylor series of the nuclear coordinates, taking into account the full symmetry of the investigated system. The diabatic potential matrix is connected to the adiabatic one by the unitary transformation that diagonalizes the diabatic matrix. This approach is based on the seminal work by Jahn and Teller, ${ }^{11}$ who found that the splitting of degenerate electronic states of 
(nonlinear) molecules is due to vibronic coupling. Particularly the vibronic coupling approach which includes only the first nonvanishing coupling terms is widely used, e.g., by Köppel, Domcke, and Cederbaum, ${ }^{12}$ to study the nuclear dynamics of systems with coupled electronic states. A significant advantage of such a diabatization is that only the adiabatic state energies are needed but no explicit information about the electronic wave functions is necessary. In practice the parameters of the diabatic matrix are determined by a least-squares fitting procedure of its eigenvalues to the adiabatic energies which can be calculated by standard $a b$ initio electronic structure methods.

In this work we present a study of the effect of higher order Jahn-Teller coupling terms. In particular we investigate the case when the potential energy surfaces of the degenerate state show pronounced anharmonicity. To this end the fully coupled diabatic potential matrix for a general $D_{3 h}$ or $C_{3 v} E \otimes e$ system is derived as a Taylor expansion up to sixth order. This sixth order potential matrix is compared to JahnTeller expansions of lower order. We also test different approximations which are based on the well known second order Jahn-Teller Hamiltonian which is augmented by higher order terms on the diagonal only (in real representation) to account for anharmonicity.

This paper is organized as follows: In Sec. II the higher order Jahn-Teller terms are derived by symmetry considerations. The corresponding potential matrices are given in both complex and real representations. The different JahnTeller orders are tested in Sec. III, where wave-packet propagations allow the direct comparison of the dynamics on these models. Similarly, models approximating the Jahn-Teller expansions are tested in Sec. IV. Our findings and conclusions will be briefly discussed and summarized in Sec. V.

\section{HIGHER ORDER JAHN-TELLER COUPLINGS}

\section{A. Symmetry}

In this section the Hamiltonian matrix for the wellknown $E \otimes e$ Jahn-Teller system up to sixth order will be developed. In this case a doubly degenerate electronic state is coupled by a set of nuclear coordinates belonging to a degenerate vibrational mode. This situation can arise for all molecules with a symmetry point group containing an $n$-fold axis with $n \geqslant 3$. A particularly simple example is a general $\mathrm{AB}_{3}$ molecule for which the global potential energy function (and thus the Hamiltonian) necessarily is of $D_{3 h}$ symmetry. In Sec. III we will use the $\mathrm{NO}_{3}$ radical as an example. It has a planar $D_{3 h}$ ground state geometry and low-lying excited electronic states of ${ }^{2} E^{\prime \prime}$ and ${ }^{2} E^{\prime}$ symmetry. However, the following symmetry considerations and obtained expressions are generally valid for any $E \otimes e$ system containing a $C_{3}$ rotation axis.

The $D_{3 h}$ group contains as symmetry operators besides the trivial unit operator $(\hat{E})$ a rotation by $2 \pi / 3\left(\hat{C}_{3}\right)$, three rotations by $\pi / 2\left(\hat{C}_{2}\right)$, three reflections on mirror planes intersecting along the $C_{3}$ axis $\left(\hat{\sigma}_{v}\right)$, and the reflection on the plane perpendicular to the $C_{3}$ axis $\left(\hat{\sigma}_{h}\right)$. The irreducible representations $\Gamma_{i}$ of the group are $A_{1}^{\prime}, A_{2}^{\prime}, E^{\prime}, A_{1}^{\prime \prime}, A_{2}^{\prime \prime}$, and $E^{\prime \prime}$ but the six dynamic variables which remain after separa- tion of overall rotation $\left(a_{2}^{\prime}\right.$ and $\left.e^{\prime \prime}\right)$ and translation $\left(a_{1}^{\prime \prime}\right.$ and $\left.e^{\prime}\right)$ can only transform like $a_{1}^{\prime}, e^{\prime}$, and $a_{2}^{\prime \prime}$ for the $\mathrm{AB}_{3}$ system (it is customary to label the $\Gamma_{i}$ 's for coordinates by small and for electronic states by capital letters). More precisely, two of the internal motions of the $\mathrm{AB}_{3}$ system transform as $e^{\prime}$ (asymmetric stretching and bending) and the remaining internal degrees of freedom transform as $a_{1}^{\prime}$ (symmetric stretching) and $a_{2}^{\prime \prime}$ ("umbrella"). Thus the degeneracy of both $E^{\prime}$ and $E^{\prime \prime}$ electronic states is lifted by two $e^{\prime}$ motions each. The degeneracy of the states means that there exist two linearly independent eigenvectors to the same eigenvalue of the electronic Hamiltonian, labeled $\left|\Psi_{x}\right\rangle$ and $\left|\Psi_{y}\right\rangle$, which span a two-dimensional subspace. Every normalized linear combination (vector of this subspace) will also be an eigenfunction to the same eigenvalue. Thus we have the freedom to perform arbitrary unitary transformations on such vectors. The same is true for the twodimensional subspace formed by the components $x$ and $y$ of the $e^{\prime}$ modes. Exactly this will be utilized in the following derivation of the Jahn-Teller Hamiltonian for an $E \otimes e$ coupling of an arbitrary $\mathrm{AB}_{3}$ system. This approach uses the ideas of Longuet-Higgins about the dependence of the electronic wave function on the nuclear coordinates. ${ }^{13}$

\section{B. Selection rules in complex representation}

The total Hamiltonian $\hat{H}$ must be invariant under the symmetry operations $\hat{S}$ of the nuclear point group since $[\hat{H}, \hat{S}]=0$. Now we wish to express the potential energies used in $\hat{H}$, which are determined by the eigenvalues of the electronic Hamiltonian $\hat{H}_{\mathrm{el}}$ at given nuclear geometries, by a Taylor expansion in the nuclear coordinates. The invariance condition allows the straightforward derivation of the nonvanishing terms in this expansion. To this end it is beneficial to transform the nuclear coordinates belonging to the degenerate mode and the eigenvectors of the degenerate electronic state to the complex representation by the unitary transformation $\mathbf{U}^{\dagger}$,

$$
\sqrt{2} \mathbf{U}^{\dagger}\left(\begin{array}{l}
x \\
y
\end{array}\right)=\left(\begin{array}{cc}
1 & i \\
1 & -i
\end{array}\right)\left(\begin{array}{l}
x \\
y
\end{array}\right)=\left(\begin{array}{l}
x+i y \\
x-i y
\end{array}\right)=\left(\begin{array}{l}
Q_{+} \\
Q_{-}
\end{array}\right)
$$

and

$$
\begin{aligned}
\mathbf{U}^{\dagger} \boldsymbol{\Psi}_{(x y)} & =\mathbf{U}^{\dagger}\left(\begin{array}{l}
\left\langle\Psi_{x}\right| \\
\left\langle\Psi_{y}\right|
\end{array}\right)=\frac{1}{\sqrt{2}}\left(\begin{array}{l}
\left\langle\Psi_{x}\right|+i\left\langle\Psi_{y}\right| \\
\left\langle\Psi_{x}\right|-i\left\langle\Psi_{y}\right|
\end{array}\right)=\left(\begin{array}{c}
\left\langle\Psi_{+}\right| \\
\left\langle\Psi_{-}\right|
\end{array}\right) \\
& =\Psi_{ \pm}
\end{aligned}
$$

In the complex representation the coordinates $Q_{+}$and $Q_{-}$ and the state functions $\left\langle\Psi_{+}\right|$and $\left\langle\Psi_{-}\right|$are eigenfunctions of the symmetry operator $\hat{C}_{3}$ (rotation by $2 \pi / 3$ ) with eigenvalues $e^{ \pm 2 \pi i / 3}$. Thus, a rotation of $2 \pi / 3$ transforms the complex coordinates as 
TABLE I. Nonvanishing terms of the Hamiltonian matrix in complex representation.

\begin{tabular}{lcc}
\hline \hline Order & Diagonal $H_{++}=H_{--}$ & Off-diagonal $H_{+-}=\left(H_{-+}\right)^{*}$ \\
\hline 0 & $Q_{+}^{0} Q_{-}^{0}$ & - \\
1 & - & $Q_{+}^{0} Q_{-}^{1}$ \\
2 & $Q_{+}^{1} Q_{-}^{1}$ & $Q_{+}^{2} Q_{-}^{0}$ \\
3 & $Q_{+}^{3} Q_{-}^{0}$ and $Q_{+}^{0} Q_{-}^{3}$ & $Q_{+}^{1} Q_{-}^{2}$ \\
4 & $Q_{+}^{2} Q_{-}^{2}$ & $Q_{+}^{0} Q_{-}^{4}$ and $Q_{+}^{3} Q_{-}^{1}$ \\
5 & $Q_{+}^{4} Q_{-}^{1}$ and $Q_{+}^{1} Q_{-}^{4}$ & $Q_{+}^{2} Q_{-}^{3}$ and $Q_{+}^{5} Q_{-}^{0}$ \\
6 & $Q_{+}^{6} Q_{-}^{0}$ and $Q_{+}^{3} Q_{-}^{3}$ and $Q_{+}^{0} Q_{-}^{6}$ & $Q_{+}^{1} Q_{-}^{5}$ and $Q_{+}^{4} Q_{-}^{2}$ \\
\hline \hline
\end{tabular}

$$
\hat{C}_{3} Q_{+}=e^{+2 \pi i / 3} Q_{+} \quad \text { and } \hat{C}_{3} Q_{-}=e^{-2 \pi i / 3} Q_{-} .
$$

The electronic wave functions are transformed accordingly by the rotation as

$$
\begin{aligned}
& \hat{C}_{3}\left\langle\Psi_{+}\right|=e^{+2 \pi i / 3}\left\langle\Psi_{+}\right| \text {and } \hat{C}_{3}\left\langle\Psi_{-}\right|=e^{-2 \pi i / 3}\left\langle\Psi_{-}\right| \\
& \hat{C}_{3}\left|\Psi_{+}\right\rangle=e^{-2 \pi i / 3}\left|\Psi_{+}\right\rangle \text {and } \hat{C}_{3}\left|\Psi_{-}\right\rangle=e^{+2 \pi i / 3}\left|\Psi_{-}\right\rangle .
\end{aligned}
$$

The representation of the electronic Hamiltonian in the $\left\{\left|\Psi_{+}\right\rangle,\left|\Psi_{-}\right\rangle\right\}$basis is given by

$$
\hat{H}_{\mathrm{el}}=\boldsymbol{\Psi}_{ \pm}^{\dagger} \mathbf{H}_{ \pm} \boldsymbol{\Psi}_{ \pm}=\sum_{i, j}\left|\Psi_{i}\right\rangle H_{i j}\left\langle\Psi_{j}\right| \quad(i, j=+,-)
$$

and the matrix elements $H_{i j}=\left\langle\Psi_{i}\left|\hat{H}_{\mathrm{el}}\right| \Psi_{j}\right\rangle(i, j=+,-)$ are expanded in Taylor series up to sixth order in $Q_{+}, Q_{-}$. For example, $H_{++}$is expanded as

$$
H_{++}=\sum_{p+q=0}^{6} \frac{c_{p, q}^{(+)}}{(p+q) !} Q_{+}^{p} Q_{-}^{q} .
$$

Each term in the expansion Eq. (6) has to fulfill the invariance condition under the symmetry operations and therefore most of the expansion coefficients are zero. For example,

$$
\begin{aligned}
\hat{C}_{3}\left|\Psi_{+}\right\rangle Q_{+}^{p} Q_{-}^{q}\left\langle\Psi_{+}\right|= & e^{-2 \pi i / 3} e^{(+p) 2 \pi i / 3} e^{(-q) 2 \pi i / 3} e^{2 \pi i / 3} \\
& \times\left|\Psi_{+}\right\rangle Q_{+}^{p} Q_{-}^{q}\left\langle\Psi_{+}\right|
\end{aligned}
$$

can contribute only for $(p, q)$ combinations which fulfill the condition $(p-q) \bmod (3)=0$ because only in this case the phase factor of Eq. (7) is unity. The same procedure is repeated for the remaining elements of the Hamiltonian [Eq. (5)], resulting in ten nonvanishing diagonal and nine nonvanishing off-diagonal terms. These terms are summarized in Table I.

Finally, it needs to be verified that the surviving terms also fulfill the invariance condition for all other symmetry operators belonging to the nuclear point group. However, it is easier to do this in the real representation of coordinates and wave functions since $x, y$ and $\left\langle\Psi_{x}\right|,\left\langle\Psi_{y}\right|$ are eigenfunctions of $\hat{C}_{2}, \hat{\sigma}_{v}$, and $\hat{\sigma}_{h}$.

\section{Hamiltonian in real representation}

The coordinates of choice for the dynamics treatment as well as for the $a b$ initio computation of electronic energies are the real variables $x$ and $y$. The matrix representation $\mathcal{H}$ of the electronic Hamiltonian in the real representation, including all Jahn-Teller couplings up to sixth order, is obtained by the back transformation

$$
\hat{H}_{\mathrm{el}}=\boldsymbol{\Psi}_{ \pm}^{\dagger} \mathbf{U}^{\dagger} \mathbf{U} \mathbf{H}_{ \pm} \mathbf{U}^{\dagger} \mathbf{U} \boldsymbol{\Psi}_{ \pm}=\boldsymbol{\Psi}_{(x y)}^{\dagger} \mathcal{H} \boldsymbol{\Psi}_{(x y)} .
$$

This leads to the factorized expression

$$
\begin{aligned}
\mathcal{H} & =\mathbf{U H}_{ \pm} \mathbf{U}^{\dagger} \\
& =\sum_{n=0}^{6} \frac{1}{n !}\left\{\left(\begin{array}{cc}
\mathcal{V}^{(n)} & 0 \\
0 & \mathcal{V}^{(n)}
\end{array}\right)+\left(\begin{array}{cc}
\mathcal{W}^{(n)} & \mathcal{Z}^{(n)} \\
\mathcal{Z}^{(n)} & -\mathcal{W}^{(n)}
\end{array}\right)\right\}
\end{aligned}
$$

for the potential matrix $\mathcal{H}$ where $\mathcal{V}^{(n)}$ contains the diagonal elements [Eqs. (10)], corresponding to the potential in the absence of the Jahn-Teller effect, $\mathcal{W}^{(n)}$ the diagonal, and $\mathcal{Z}^{(n)}$ the off-diagonal coupling elements [Eqs. (11) and (12)] for each order $n$ in the real representation. The matrix elements of $\mathcal{V}, \mathcal{W}$, and $\mathcal{Z}$ up to sixth order are given by

$$
\begin{aligned}
& \mathcal{V}^{(0)}=a_{1}^{(0)}, \\
& \mathcal{V}^{(1)}=0 \\
& \mathcal{V}^{(2)}=a_{1}^{(2)}\left[x^{2}+y^{2}\right] \text {, } \\
& \mathcal{V}^{(3)}=a_{1}^{(3)}\left[2 x^{3}-6 x y^{2}\right] \\
& \mathcal{V}^{(4)}=a_{1}^{(4)}\left[x^{4}+2 x^{2} y^{2}+y^{4}\right], \\
& \mathcal{V}^{(5)}=a_{1}^{(5)}\left[2 x^{5}-4 x^{3} y^{2}-6 x y^{4}\right], \\
& \mathcal{V}^{(6)}=a_{1}^{(6)}\left[2 x^{6}-30 x^{4} y^{2}+30 x^{2} y^{4}-2 y^{6}\right] \\
& +a_{2}^{(6)}\left[x^{6}+3 x^{4} y^{2}+3 x^{2} y^{4}+y^{6}\right] \text {, } \\
& \mathcal{W}^{(0)}=0 \text {, } \\
& \mathcal{W}^{(1)}=\lambda_{1}^{(1)} x \\
& \mathcal{W}^{(2)}=\lambda_{1}^{(2)}\left[x^{2}-y^{2}\right], \\
& \mathcal{W}^{(3)}=\lambda_{1}^{(3)}\left[x^{3}+x y^{2}\right], \\
& \mathcal{W}^{(4)}=\lambda_{1}^{(4)}\left[x^{4}-6 x^{2} y^{2}+y^{4}\right]+\lambda_{2}^{(4)}\left[x^{4}-y^{4}\right] \text {, } \\
& \mathcal{W}^{(5)}=\lambda_{1}^{(5)}\left[x^{5}-10 x^{3} y^{2}+5 x y^{4}\right] \\
& +\lambda_{2}^{(5)}\left[x^{5}+2 x^{3} y^{2}+x y^{4}\right] \text {, } \\
& \mathcal{W}^{(6)}=\lambda_{1}^{(6)}\left[x^{6}-5 x^{4} y^{2}-5 x^{2} y^{4}+y^{6}\right] \\
& +\lambda_{2}^{(6)}\left[x^{6}+x^{4} y^{2}-x^{2} y^{4}-y^{6}\right] \text {, } \\
& \mathcal{Z}^{(0)}=0 \\
& \mathcal{Z}^{(1)}=\lambda_{1}^{(1)} y, \\
& \mathcal{Z}^{(2)}=-2 \lambda_{1}^{(2)} x y
\end{aligned}
$$




$$
\begin{aligned}
\mathcal{Z}^{(3)}= & \lambda_{1}^{(3)}\left[x^{2} y+y^{3}\right], \\
\mathcal{Z}^{(4)}= & \lambda_{1}^{(4)}\left[4 x^{3} y-4 x y^{3}\right]+\lambda_{2}^{(4)}\left[-2 x^{3} y-2 x y^{3}\right], \\
\mathcal{Z}^{(5)}= & \lambda_{1}^{(5)}\left[-5 x^{4} y+10 x^{2} y^{3}-y^{5}\right] \\
& +\lambda_{2}^{(5)}\left[x^{4} y+2 x^{2} y^{3}+y^{5}\right] \\
\mathcal{Z}^{(6)}= & \lambda_{1}^{(6)}\left[4 x^{5} y-4 x y^{5}\right] \\
& +\lambda_{2}^{(6)}\left[-2 x^{5} y-4 x^{3} y^{3}-2 x y^{5}\right] .
\end{aligned}
$$

It remains to be tested whether the derived Hamiltonian matrix also fulfills the invariance condition with respect to the $\hat{C}_{2}, \hat{\sigma}_{v}$, and $\hat{\sigma}_{h}$ operations. It is found that $x$ is an eigenfunction with eigenvalue +1 with respect to all operators. In contrast $y$ has eigenvalues of -1 for $\hat{C}_{2}$ and $\hat{\sigma}_{v}$ but +1 for $\hat{\sigma}_{h}$. The eigenvalues corresponding to $\left|\Psi_{x}\right\rangle$ and $\left|\Psi_{y}\right\rangle$ depend on the actual electronic state. For a state of $E^{\prime}$ symmetry the action of the operators are as follows: $\hat{\sigma}_{h}$ results in eigenvalues +1 for both components while the other operators yield +1 for $\left|\Psi_{x}\right\rangle$ and -1 for $\left|\Psi_{y}\right\rangle$. For an $E^{\prime \prime}$ state only the action of $\hat{\sigma}_{h}$ is different because it gives eigenvalues of -1 for both state components. With these properties the invariance condition is verified analog to Eq. (7), using the real representation for the matrix elements and coordinates. As a result all the diagonal terms [Eqs. (10) and (11)] can only contain even powers of $y$ while the off-diagonal terms [Eqs. (12)] must only have odd powers of $y$. Due to the form of Eq. (7) these rules are independent of the transformation properties of the electronic wave function with respect to $\hat{\sigma}_{h}$ and are thus the same for $E^{\prime}$ and $E^{\prime \prime}$ states. It is easily verified that the derived Hamiltonian follows these rules and is thus invariant under all symmetry operations of the $D_{3 h}$ group.

\section{APPLICATION: A COMPARATIVE STUDY}

\section{A. $n$th order Jahn-Teller models (JTn)}

The effect of using the expansion presented in Sec. II is tested on a two-dimensional $E \otimes e$ Jahn-Teller model system which represents the $e^{\prime}$ stretching vibration of the photoactive ${ }^{2} E^{\prime}$ state of $\mathrm{NO}_{3}$. The necessary data to determine the Hamiltonian matrix parameters are obtained from high quality ab initio MR-SDCI calculations. To this end first the Cartesian normal mode coordinates of the electronic ground state $\left({ }^{2} A_{2}^{\prime}\right)$ were determined. ${ }^{14}$ Then cuts along the $x$ and $y$ components of the asymmetric $e^{\prime}$ stretching vibration were calculated for both the ${ }^{2} E_{x}^{\prime}$ and ${ }^{2} E_{y}^{\prime}$ components of the second excited state. The same electronic structure methods were used as described in detail in Ref. 15. The degeneracy is lifted along both $x$ and $y$ as expected for a Jahn-Teller coupling case. This particular system was chosen as a test model because of the pronounced anharmonicity of the ${ }^{2} E^{\prime}$ state of $\mathrm{NO}_{3}$, especially along the $x$ component. In this case a vibronic coupling approach ${ }^{12}$ limited to the first nonvanishing coupling terms and to the harmonic approximation cannot be expected to be very reliable.

One feasible solution to account for the anharmonicity is to extend the Jahn-Teller model to higher order as explained
TABLE II. Parameters for the JT6 model in (a.u.).

\begin{tabular}{lccrc}
\hline \hline$n$ & $a_{1}^{(n)}$ & $a_{2}^{(n)}$ & $\lambda_{1}^{(n)}$ & $\lambda_{2}^{(n)}$ \\
\hline 0 & $7.411 \times 10^{-02}$ & - & - & - \\
1 & - & - & $2.121 \times 10^{-04}$ & - \\
2 & $4.046 \times 10^{-05}$ & - & $-8.422 \times 10^{-06}$ & - \\
3 & $-2.626 \times 10^{-07}$ & - & $-2.948 \times 10^{-07}$ & - \\
4 & $2.264 \times 10^{-08}$ & - & $-2.322 \times 10^{-09}$ & $3.270 \times 10^{-09}$ \\
5 & $-7.901 \times 10^{-10}$ & - & $-4.018 \times 10^{-11}$ & $1.544 \times 10^{-10}$ \\
6 & $-6.701 \times 10^{-14}$ & $1.151 \times 10^{-11}$ & $6.512 \times 10^{-12}$ & $4.626 \times 10^{-12}$ \\
\hline \hline
\end{tabular}

in Sec. II. However, though the treatment of the Jahn-Teller effect is well studied in general, ${ }^{16,17}$ to the best of our knowledge the present study is the first example to extend the Jahn-Teller expansion beyond third order. Alternatively, the so-called regularized diabatization could be applied as described in Ref. 10. This method is not limited to any particular functional form of the adiabatic or diabatic potentials but can only be applied if the region of interest encompasses only a single conical intersection.

In order to apply the formalism presented in Sec. II to the $e^{\prime}$ stretching vibration of the ${ }^{2} E^{\prime}$ state of $\mathrm{NO}_{3}$, the expansion coefficients of the diabatic potential matrix [Eqs. (10)-(12)] are determined by fitting the eigenvalues of the potential matrix [Eq. (9)] to the $a b$ initio results, using a Levenberg-Marquardt least squares procedure. Thus, both components of the state are fitted simultaneously along both coordinates. The root mean-squares error (rms) of this fit is $6.25 \cdot 10^{-3} \mathrm{eV}$ which shows that the applied JT-Hamiltonian can represent the $a b$ initio data very well. Furthermore it is known that for $\mathrm{NO}_{3}$ there is also strong pseudo-Jahn-Teller (PJT) coupling present between the ${ }^{2} A_{2}^{\prime}$ ground and the ${ }^{2} E^{\prime}$ state treated here. ${ }^{18}$ Thus it is a reasonable assumption that the remaining error is due to a large extent to the neglect of this additional coupling. The inclusion of the PJT coupling effect will be necessary for a correct treatment of the physical system $\mathrm{NO}_{3}$ and is easily achieved following the techniques demonstrated in Sec. II. However, for the present model study it has been omitted because it would only obscure matters. By excluding the PJT effects a reference model for the pure JT coupling is obtained which is the subject of this study. Thus, it should be kept in mind that this model does not aim for a perfect description of the physics of the $\mathrm{NO}_{3}$ system. In the following this model, including all Jahn-Teller coupling terms up to sixth order, will be referred to as "JT6." Table II gives the numerical values of the parameters for the JT6 model.

The quality of the fits for lower order Jahn-Teller Hamiltonians, denoted by JT2-JT5 and containing only terms of orders up to $n_{\max }$, was studied by fitting these models with respect to data points obtained by the JT6 Hamiltonian taken as a reference. The model data was chosen as reference mainly in order to test the effect of the diabatization scheme rather than to study the quality of the fit to the physical system $\mathrm{NO}_{3}$. Different fits were performed which are distinguished by the different excess energies of data points included in the fitting data. In this way information is obtained about the energy range in which the respective model can be expected to yield reliable quantitative results. Table III pre- 
TABLE III. Errors in (eV) for different excess energies of lower-order JahnTeller Hamiltonians (JT2-JT5) with respect to the JT6 model.

\begin{tabular}{|c|c|c|c|c|c|c|c|c|}
\hline \multirow{2}{*}{$\begin{array}{l}\text { Exc. } \\
\text { En. } \\
(\mathrm{eV})\end{array}$} & \multicolumn{2}{|c|}{ JT2 } & \multicolumn{2}{|c|}{ JT3 } & \multicolumn{2}{|c|}{ JT4 } & \multicolumn{2}{|c|}{ JT5 } \\
\hline & $\max$ & $\mathrm{rms}$ & max. & $\mathrm{rms}$ & $\max$ & $\mathrm{rms}$ & $\max$ & $\mathrm{rms}$ \\
\hline 1.0 & 0.1974 & 0.0549 & 0.0285 & 0.0105 & 0.0040 & 0.0015 & 0.0002 & 0.0001 \\
\hline 2.0 & 0.5181 & 0.1722 & 0.0990 & 0.0360 & 0.0182 & 0.0067 & 0.0022 & 0.0008 \\
\hline 3.0 & 0.7439 & 0.2364 & 0.1808 & 0.0562 & 0.0401 & 0.0139 & 0.0036 & 0.0013 \\
\hline 4.0 & 1.0969 & 0.3306 & 0.2746 & 0.0816 & 0.0623 & 0.0217 & 0.0059 & 0.0021 \\
\hline
\end{tabular}

sents the dependence of the errors (maximum absolute and rms errors), computed for the respective fitting data along the $x$ and $y$ cuts, with respect to both the highest order kept in the expansion of the potential matrix [Eqs. (10)-(12)] and the excess energy of the reference points taken into account for the fitting. An exponential decrease of the errors is observed as a function of the order $n_{\max }$ for all excess energies tested. The dependence of the errors as a function of the excess energy is found to be nearly linear, decreasing as the excess energy decreases. This trend is easily explained by the fact that the anharmonicity of the potential surfaces depends directly on the energy range considered. From the values of the rms errors obtained for this particular system it can be expected that models including all terms up to order four and higher will give very similar results for processes which involve only small excess energies. For example, the rms of the JT4 potential is $<1 \%$ of the excess energy if only points below $2 \mathrm{eV}$ are considered. However, for processes that sample high energy regions of the potential even with JT4 and JT5 noticeable differences may result.

\section{B. Potential energy surfaces}

Cuts of the adiabatic potential energy surfaces along $x$ are presented in Fig. 1 for the reference JT6, the lower order JT2-JT4 models. The curves of the JT5 model along both $x$ and $y$ are indistinguishable from the JT6 curves on the scale of the figure and thus are not depicted. The reference JT6 potentials are indicated by solid lines whereas dashed lines and open circles have been used for the lower order models. If not mentioned otherwise, the values of the parameters used are the ones obtained by fitting all data including points up to $4 \mathrm{eV}$ of excess energy. All parameter values except for the JT6 reference model are given in the Appendix. The errors given in Table III also become apparent in Fig. 1. It is clearly seen that even at very low energies the JT2 potentials give a very poor representation of the reference curves and at higher energies the deviations become fairly disastrous. In contrast, the JT3 curves resemble the reference data much better and differ only moderately within the depicted range of $x$. The deviations of the JT4 potentials are so small that they are hardly visible in the figure. However, for energies much higher than $6 \mathrm{eV}$ (i.e., excess energy $>4 \mathrm{eV}$ ) the differences are still significant. We note that the $a b$ initio cuts are also indistinguishable from the JT6 curves on this scale. Inspection of the potential cuts along $y$ result in similar conclusions.

Figure 2 presents the cuts along the $y$ coordinate of the diabatic coupling term (off-diagonal matrix element) for the
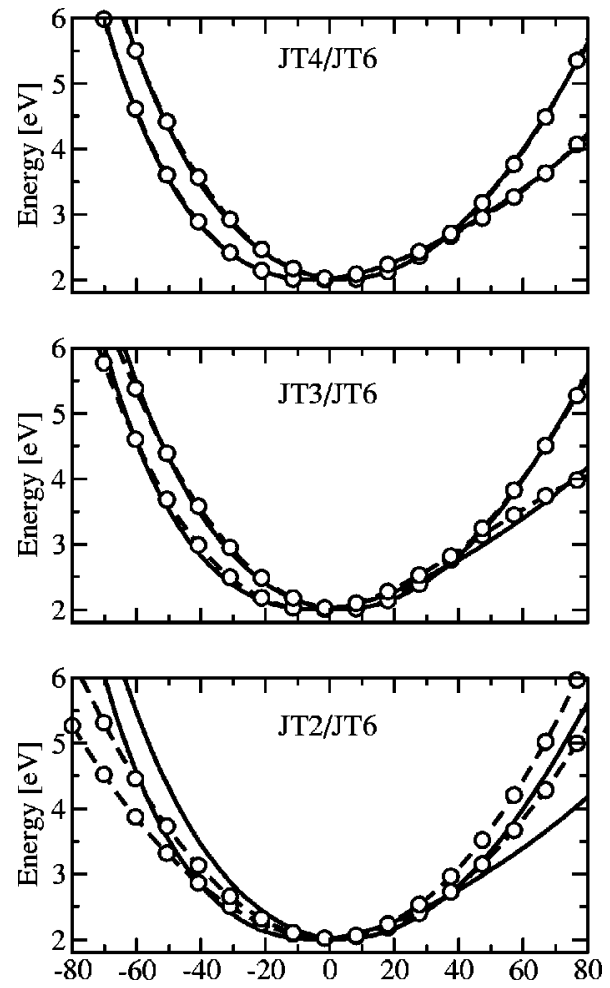

FIG. 1. Adiabatic potential energy surfaces in $\mathrm{eV}$ along $x$ coordinate for the models JT2, JT3, and JT4 (dashed lines with open circles) compared to the JT6 reference model (solid lines).

different orders of the Jahn-Teller expansion. The reference JT6 data is indicated by a solid line whereas the lower order models are depicted as dashed lines with open symbols. Only the coupling along $y$ is depicted because the coupling along $x$ is identically zero due to symmetry. From Fig. 2 it becomes apparent that the diabatic coupling shows a strongly nonlinear behavior in the coordinate range investigated. In stark contrast the coupling of the well-known JT2 model gives a straight line because the coupling is linear in $y$ for this cut. The coupling of the reference JT6 Hamiltonian is roughly linear between \pm 25 a.u. But even in this range the JT2 is

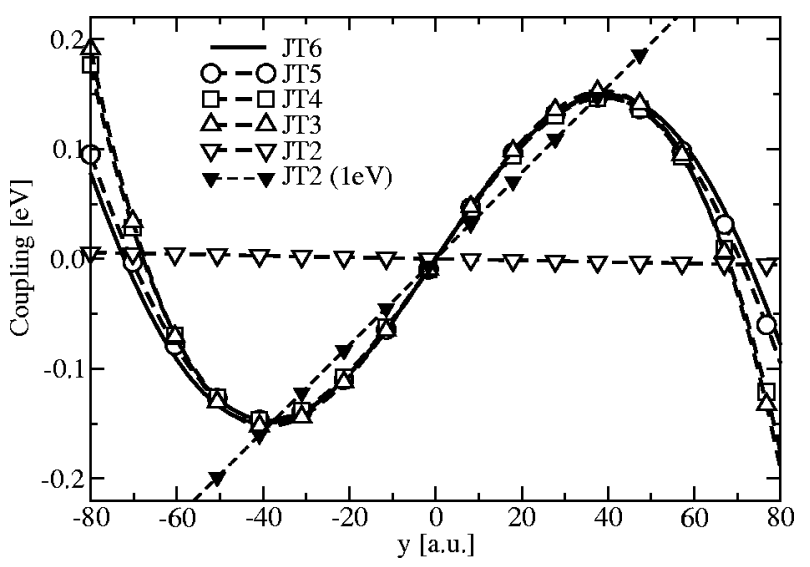

FIG. 2. Diabatic coupling matrix elements in $\mathrm{eV}$ along $y$ for the models JT2-JT5 (dashed lines with open symbols) with respect to the reference model JT6 (solid line). The coupling matrix element for JT2 obtained with an excess energy criterion of only $1 \mathrm{eV}$ is presented as a dashed line with filled triangles. 
quantitatively inaccurate as becomes obvious from the different slopes at the origin. An improvement of the JT2 is seen when using a lower energy criterion for the fitting procedure. The slope corresponding to the fit including only data points up to $1 \mathrm{eV}$ excess energy (dashed line with filled triangles) is in significantly better agreement with the reference but still far from being a good approximation to JT6. The range of \pm 25 a.u. is found to be smaller than the width of the initial vibrational ground state wave function. Thus the wave packet will definitely sample a configuration space extending into a region where the coupling is certainly not linear anymore. This indicates that JT2 can hardly be a very reliable model for the nonadiabatic nuclear dynamics of such a system.

Going only one order higher in the Jahn-Teller expansion (JT3) results in at least roughly the correct shape of the coupling curve. The first noticeable deviation occurs around \pm 50 a.u. which corresponds to an excess energy of about 0.8 $\mathrm{eV}$. However, beyond 50 a.u. the deviations from the JT6 reference quickly become considerable and will affect the dynamics calculations. The same is true for the JT4 expansion. Only the JT5 results are in very close agreement with the reference model over the range presented.

\section{Vertical excitation}

In order to test the effect of the applied diabatization scheme on the nuclear dynamics wave packet propagations are performed with the different potentials obtained. The first test is a vertical excitation starting from a Gaussian fit of the vibrational ground state of the electronic ground state of $\mathrm{NO}_{3} .{ }^{19}$ The Gaussian fit is determined by propagation in imaginary time on the ground state potential of $\mathrm{NO}_{3}$. This initial packet thus has a physical width and a sampling of the relevant configuration space is expected during the propagation on the excited states surfaces. A Franck-Condon excitation is assumed and the wave packet is put on one of the diabatic surfaces. The propagation of this 2-mode wavepacket on 2 coupled electronic states is performed by a standard propagation scheme using Fourier transformation.

A sensitive measure of the dynamics is the calculated autocorrelation function which is presented in Fig. 3 for the reference JT6 model as well as for the lower order models JT2-JT5. The parameters used for the lower order models are determined from the fit with $4 \mathrm{eV}$ excess energy (cf. Table III). Additionally, the autocorrelation function for the JT2 potential fitted with $1 \mathrm{eV}$ excess energy is given. The reference autocorrelation function (JT6), depicted as a solid line, is indistinguishable from the one obtained by JT5 (dashed line with open circles). This coincidence of the JT5 and JT6 results has been observed up to 500 fs. JT4 leads to an autocorrelation function in rather good agreement with the reference model as seen in the figure. However, noticeable differences can be seen in the amplitude of the oscillations. This effect becomes more pronounced at longer time leading to an increasingly different overall envelope. The autocorrelation function obtained by JT3 deviates significantly from the reference leading to a dephasing of the curve as early as after $90 \mathrm{fs}$. The model limited to second order (fitted with data points up to $4 \mathrm{eV}$ excess energy) results in

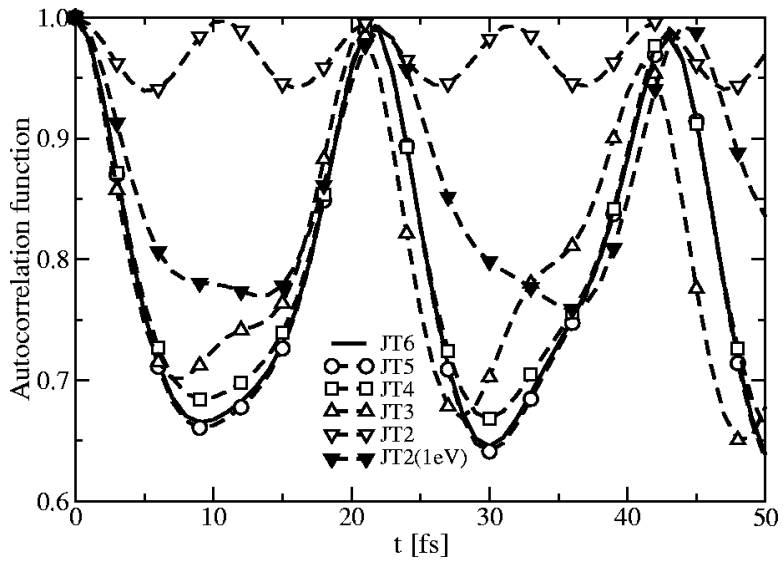

FIG. 3. Autocorrelation functions as a function of time in fs for the reference JT6 (solid line), the lower order JTn (dashed line with open symbols). The autocorrelation functions obtained for the two different JT2 Hamiltonians are presented with dashed line and full or open triangles, respectively.

an autocorrelation function completely different from the reference system with a halving of periodicity and a much smaller variation of the amplitude in the oscillations. An improvement is observed when lowering the excess energy criterion for the determination of the parameters of the model (dashed line with full triangles). Nevertheless, the corresponding autocorrelation is still significantly different from the JT6 reference.

The spectra obtained by Fourier transformation of the autocorrelation functions are depicted in Fig. 4. For ease of reading the spectra for the different models have been verti-

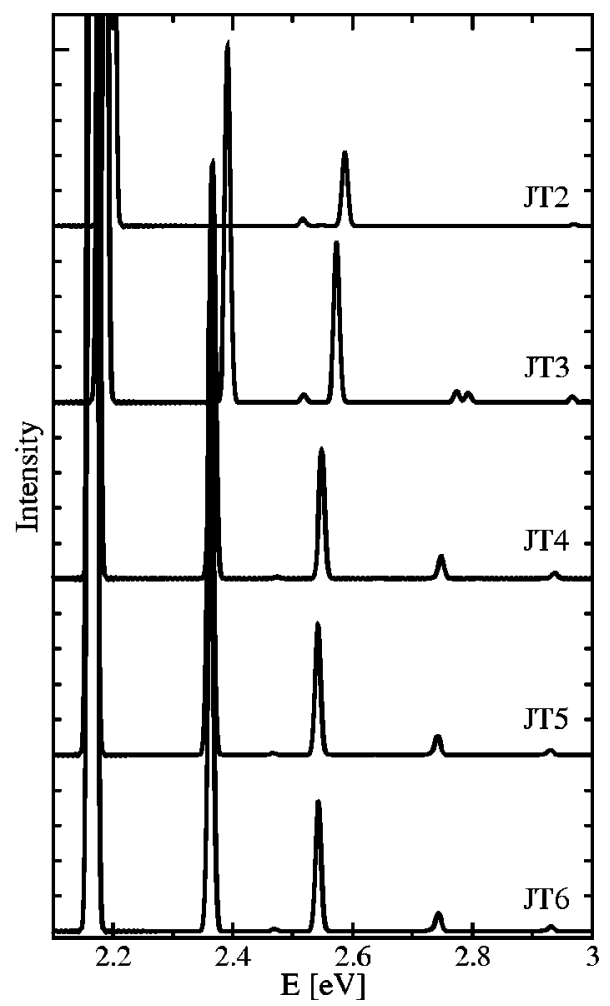

FIG. 4. Absorption spectra obtained after vertical excitation (a damping of $200 \mathrm{fs}$ has been used in the autocorrelation functions). For ease of reading, the spectra have been vertically shifted. 
cally shifted. As already concluded from the rms values (Table III) and the autocorrelation functions (Fig. 3), the spectra obtained for JT4, JT5, and JT6 are virtually identical. The spectrum obtained for JT3 displays a shift in all peaks and the one obtained for JT2 misses the second peak. Of course with a vertical excitation in this model, the number of states reachable by the wave packet is very limited but one can nevertheless see in both the autocorrelation functions and in the spectra that the dynamics on the model potentials is significantly different. A reduction of the excess energy criterion for the determination of the expansion coefficients improves the quality of the spectra for all orders since in this process only the low energy part of the surfaces is sampled by the wave packet. The differences observed from the lowering of the order in the Jahn-Teller expansion are in agreement with the conclusions drawn from the inspection of the rms values obtained from the fitting.

\section{Displaced excitation}

The coupled states represented by the Jahn-Teller expansion are not necessarily the bright states. For example the first excited ${ }^{2} E^{\prime \prime}$ state of $\mathrm{NO}_{3}$ is dipole forbidden. This state is coupled to the upper ${ }^{2} E^{\prime}$ state ${ }^{19}$ and both states intersect for displaced geometries ( $x$ and $y$ nonzero). This illustrates that a wave packet might reach such coupled states by a mechanism different to a vertical excitation from the ground state equilibrium position, e.g., through a conical intersection. Such a wave packet will sample a different configuration space compared to one that results from a vertical transition. Moreover, in the case of hot bands and two photon excitation processes the excitation does not necessarily take place for $x=0$ and $y=0$ either. This motivates the investigation of the effect of the diabatization scheme for nonvertical excitations.

This aspect of the dynamics on the model potentials is tested by the propagation of a displaced initial wave packet. The initial wave packet is taken to have the same shape as in the vertical excitation case but with a maximum located at $x=-10$ a.u. and $y=-10$ a.u. This displacement is sufficiently small to lead to wave-packet energies which only deviate slightly when using JTn, $n=2-6$. We emphasize that this propagation does not represent a physical experiment. The aim here is to show that the diabatization scheme has a stronger effect on the dynamics particularly for larger displacements from the reference geometry and at higher excess energy.

Figure 5 shows the time evolution of the mean value $\langle x\rangle$ which is related to the motion of the wave packet for the different order $n_{\max }$ used up to 100 fs. Here again JT2 and JT3 lead to a very poor description of the dynamics of this system. JT4 and JT5 show some small deviations with respect to the reference but they are still small even after $100 \mathrm{fs}$ and particularly JT5 yields almost indistinguishable results.

These results show that depending on the model the configuration space sampled by the wave packet is not identical. Since the couplings between the surfaces are a function of the coordinates, all observables related to the coupling strength will thus be different. This becomes apparent, for example, in Fig. 6 which presents the population of the lower

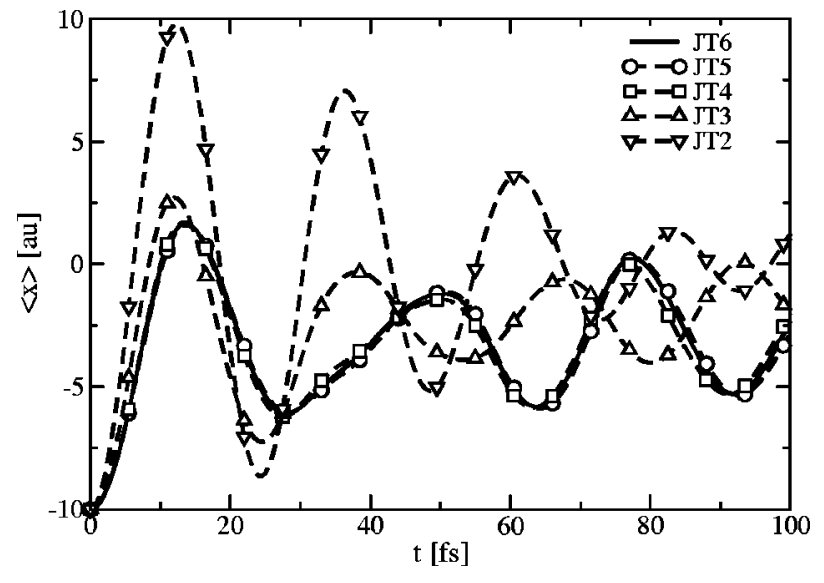

FIG. 5. Evolution of the mean value of $x$ as a function of time in fs for JT6 (full line) and lower orders (dashed line with symbols).

adiabatic state as a function of time for JT6, JT3, and JT4. As a consequence of the difference in the wave-packet location and of the difference in the coupling terms, the adiabatic populations are also different depending on the diabatization scheme chosen. JT5 (not shown here) leads to almost the same population evolution as the reference. Some differences, similar to the ones for JT3, although slightly smaller, are noticed between JT4 and the reference. JT2 (not shown here) leads to a completely different population evolution which can be improved by reducing the threshold energy criterion from 4 to $1 \mathrm{eV}$ for the determination of the parameter values.

The fact that the wave packet is not at the same position at the same time might have far reaching consequences for the treatment of realistic systems of higher dimensionality. Indeed, in realistic systems with a larger number of coordinates and electronic states, the efficiency of a transfer from one surface to another, determined by the coupling terms and by the energy separation, is an intricate function of the nuclear coordinates. It is possible that significant transfer will take place only in a rather restricted region of the configuration space. In such a system a small modification in the

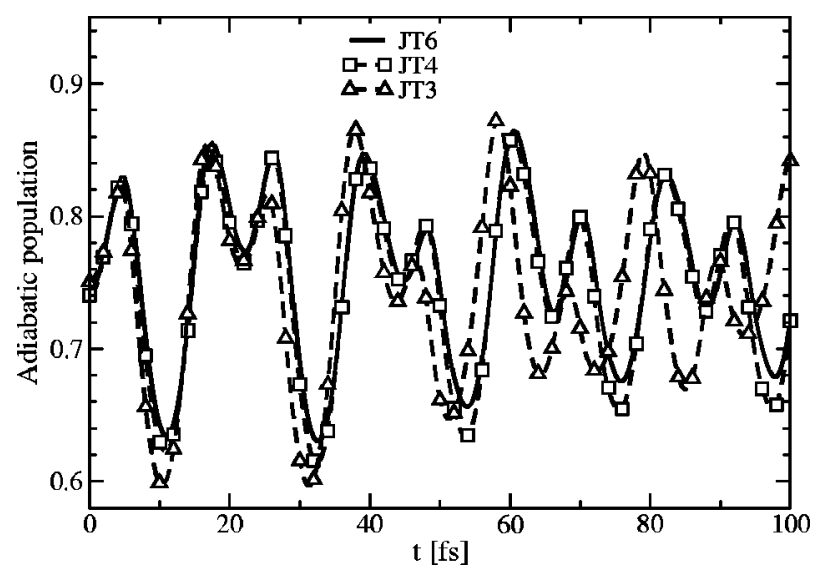

FIG. 6. Evolution of the ground state adiabatic population as a function of time in fs for the reference JT6 model (full line), for JT3 (dashed line with triangles) and for JT4 (dashed line with squares). 
TABLE IV. Parameters for the approximate model SAP6 (a.u.).

\begin{tabular}{ccccc}
\hline \hline & $a_{1}^{(0)}$ & $a_{1}^{(2)}$ & $\lambda_{1}^{(1)}$ & $\lambda_{1}^{(2)}$ \\
\hline & $7.411 \times 10^{-02}$ & $4.038 \times 10^{-05}$ & $1.850 \times 10^{-04}$ & $-6.562 \times 10^{-06}$ \\
& $\mathcal{V}_{a}^{(n)}$ & & $\mathcal{V}_{b}^{(n)}$ & \\
$n$ & $a_{1}^{(n)}$ & $a_{2}^{(n)}$ & $a_{1}^{(n)}$ & $a_{2}^{(n)}$ \\
\hline 3 & $-6.767 \times 10^{-07}$ & - & $-4.121 \times 10^{-07}$ & - \\
4 & $1.002 \times 10^{-08}$ & - & $3.780 \times 10^{-08}$ & - \\
5 & $-1.196 \times 10^{-09}$ & - & $-1.083 \times 10^{-10}$ & - \\
6 & $1.148 \times 10^{-11}$ & $5.960 \times 10^{-11}$ & $-1.313 \times 10^{-11}$ & $-5.606 \times 10^{-11}$ \\
\hline \hline
\end{tabular}

wave-packet shape and position induced by the diabatization scheme can have a dramatic effect.

The comparisons made here allow to conclude that the different diabatization schemes do have a strong influence on the dynamics. This is clearly to be expected for the lower order JTn models where the potentials are considerably different. The effects of the higher order coupling terms, besides improving the representation of the adiabatic potentials, will be assessed in the next section.

The results of the dynamics calculations indicate that expanding the Jahn-Teller series up to fourth order is sufficient to study the simple two-dimensional $E \otimes e$ model considered in this paper. Nevertheless, the authors want to point out that the effect on the dynamics has only been tested for processes strongly influenced by the lowest part of the potential energy surfaces. In cases like $\mathrm{NO}_{3}$ for which the intersection between the first excited ${ }^{2} E^{\prime \prime}$ and the upper ${ }^{2} E^{\prime}$ state occurs at strongly displaced $x$ and $y$ values, ${ }^{19}$ the restriction to the fourth order is expected to be insufficient.

\section{APPROXIMATIONS TO JT6}

\section{A. Symmetry adapted approximation (SAP6)}

In the derivation of the JTn models all possible couplings up to order $n$ are systematically included. For this reason one could consider the JTn models as a balanced description of the diagonal and off-diagonal elements of the potential matrix. The significance of the higher order coupling terms has been assessed by setting up an alternative model in which all coupling terms $\mathcal{W}^{(n)}$ and $\mathcal{Z}^{(n)}$ with $n$ $>2$ are ignored. The full Hamiltonian potential matrix of the SAPm models where $m$ is the maximum order of the expansion reads as

$$
\begin{aligned}
\mathcal{H}= & \sum_{n=0}^{2} \frac{1}{n !}\left\{\left(\begin{array}{cc}
\mathcal{V}^{(n)} & 0 \\
0 & \mathcal{V}^{(n)}
\end{array}\right)+\left(\begin{array}{cc}
\mathcal{W}^{(n)} & \mathcal{Z}^{(n)} \\
\mathcal{Z}^{(n)} & -\mathcal{W}^{(n)}
\end{array}\right)\right\} \\
& +\sum_{n=3}^{m} \frac{1}{n !}\left(\begin{array}{cc}
\mathcal{V}_{a}^{(n)} & 0 \\
0 & \mathcal{V}_{b}^{(n)}
\end{array}\right) .
\end{aligned}
$$

The matrix elements $\mathcal{V}^{(n)}, \mathcal{W}^{(n)}$, and $\mathcal{Z}^{(n)}$ are the same as given in Eqs. (10)-(12). However, to allow for the description of the different anharmonicity of the two adiabatic potential surfaces the higher order diagonal elements $\mathcal{V}_{a}^{(n)}$ and $\mathcal{V}_{b}^{(n)}$ are parametrized with different expansion coefficients. This Hamiltonian (up to sixth order), which respects the symmetry of the system contains 14 parameters, determined using the same procedure as for the lower order Jahn-Teller Hamiltonians. The corresponding numerical values are given in Table IV.

The quality of the fits for different orders of the SAPm $(m=3, \ldots, 6)$ model as well as for different excess energies has been studied in the same way as for the JTn series presented in Sec. III. Table V summarizes the dependence of the obtained maximum and rms errors. The SAP3 model results in errors comparable to JT3, although slightly larger. However, the performance of the SAP6 model is less encouraging. For lower excess energy SAP6 gives errors comparable to JT4 only, though for $4 \mathrm{eV}$ the SAP6 model performs slightly better than JT4. As for JTn, the dependence of the errors as a function of the excess energy is found to be nearly linear. The comparison of the errors with those of Table III already indicates that for the same order of the expansion, addition of higher order coupling terms leads to a better representation of the adiabatic potential surfaces. A higher order $m$ in the expansion in the SAPm models would be required to obtain errors similar to a JTn model.

While the differences in the adiabatic potentials are only quantitative, the coupling is qualitatively different between SAPm and the JT6 reference. Figure 7 presents the cut along the $y$ coordinate of the diabatic coupling term. Similar to the JT2 model, the approximative model SAP6 leads to a straight line for this cut. However, the slope at the origin is a much better approximation to the reference JT6 than the JT2 (cf. Fig. 2). Nevertheless, the off-diagonal coupling of SAP6 is incorrect for regions beyond \pm 25 a.u., where the coupling element of the reference JT6 model is not linear anymore.

Dynamical studies on the SAP6 surfaces have been performed in order to assess the significance of the different coupling patterns. For the autocorrelation function after a vertical excitation, deviations of the same magnitude as the

TABLE V. Errors in (eV) for different excess energies of different-order SAP Hamiltonians (SAP3-SAP6) and approximate model (AP6) with respect to the

\begin{tabular}{|c|c|c|c|c|c|c|c|c|c|c|}
\hline \multirow{2}{*}{$\begin{array}{l}\text { Exc. En. } \\
(\mathrm{eV})\end{array}$} & \multicolumn{2}{|c|}{ SAP3 } & \multicolumn{2}{|c|}{ SAP4 } & \multicolumn{2}{|c|}{ SAP5 } & \multicolumn{2}{|c|}{ SAP6 } & \multicolumn{2}{|c|}{ AP6 } \\
\hline & $\max$. & $\mathrm{rms}$ & $\max$ & $\mathrm{rms}$ & $\max$ & $\mathrm{rms}$ & max. & $\mathrm{rms}$ & max. & $\mathrm{rms}$ \\
\hline 1.0 & 0.0596 & 0.0194 & 0.0137 & 0.0052 & 0.0122 & 0.0048 & 0.0041 & 0.0017 & 0.0016 & 0.0005 \\
\hline 2.0 & 0.1513 & 0.0452 & 0.0335 & 0.0121 & 0.0346 & 0.0105 & 0.0097 & 0.0044 & 0.0030 & 0.0014 \\
\hline 3.0 & 0.2229 & 0.0666 & 0.0762 & 0.0225 & 0.0526 & 0.0147 & 0.0141 & 0.0064 & 0.0076 & 0.0026 \\
\hline 4.0 & 0.3244 & 0.0924 & 0.1235 & 0.0333 & 0.0712 & 0.0197 & 0.0187 & 0.0078 & 0.0098 & 0.0042 \\
\hline
\end{tabular}
JT6 model. 


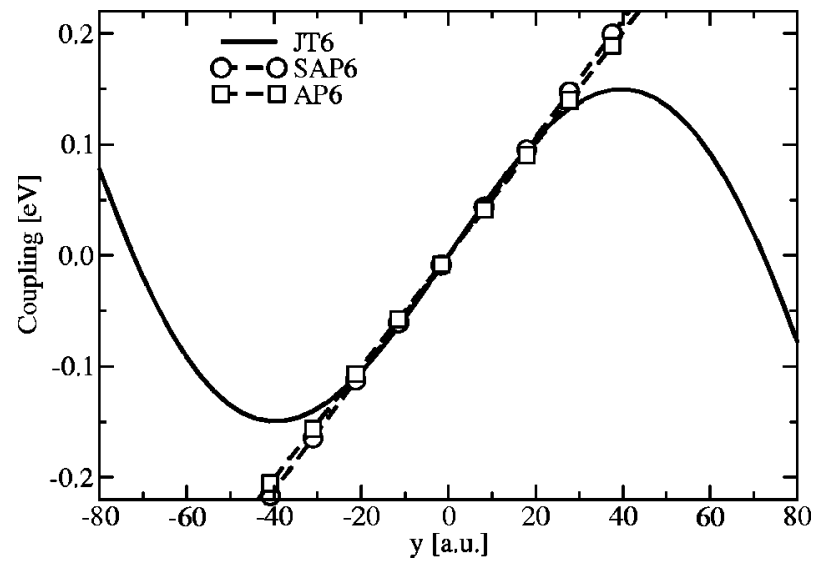

FIG. 7. Diabatic coupling matrix elements in eV along $y$ for the models SAP6 (dashed lines with open circles) and AP6 (dashed lines with open squares) with respect to the reference model JT6 (solid line).

ones seen for JT4 are observed (cf. Fig. 8). This is consistent with the conclusions drawn from the rms errors of Table V. In the corresponding spectrum, presented in Fig. 9, one can notice a slight modification of the shape of the second and third peak as well as additional low intensity features at higher energies.

The evolution of the mean value $\langle x\rangle$ after a displaced excitation is given in Fig. 10. The differences with respect to the reference model are similar to the ones observed for JT3 and much larger that those seen for JT4. This result is not obvious from the inspection of the rms errors which rank SAP6 as a better fit than JT4. It reflects the effect of the nonlinearity of the coupling which is ignored in the SAP6 model and which apparently plays a role even for rather moderately displaced excitation. Similar to the JTn series, the differences in the position and shape of the wave packet are also reflected in the adiabatic population. Like for the evolution of $\langle x\rangle$ SAP6 performs as poor as JT3 for the timedependence of the adiabatic population.

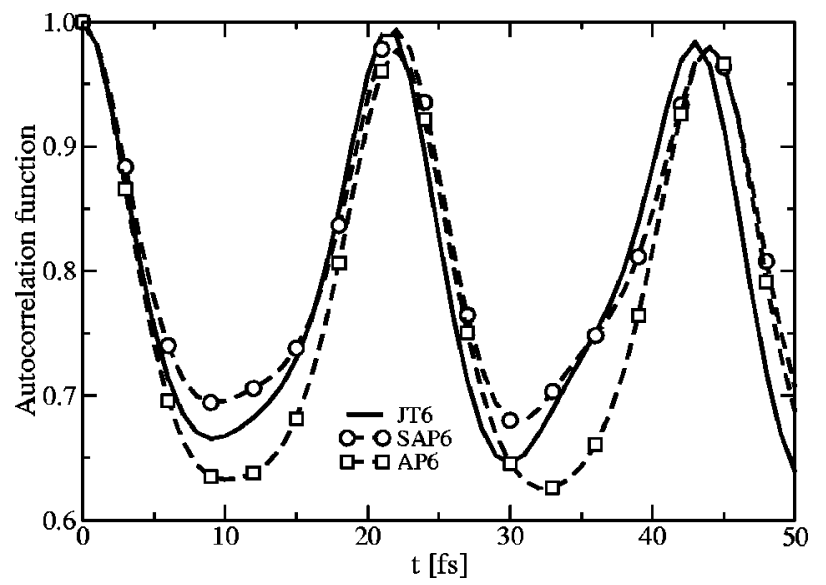

FIG. 8. Autocorrelation functions as a function of time in fs for the reference JT6 (solid line), the approximative SAP6 (dashed line with open circles) and the approximative AP6 (dashed line with open squares).

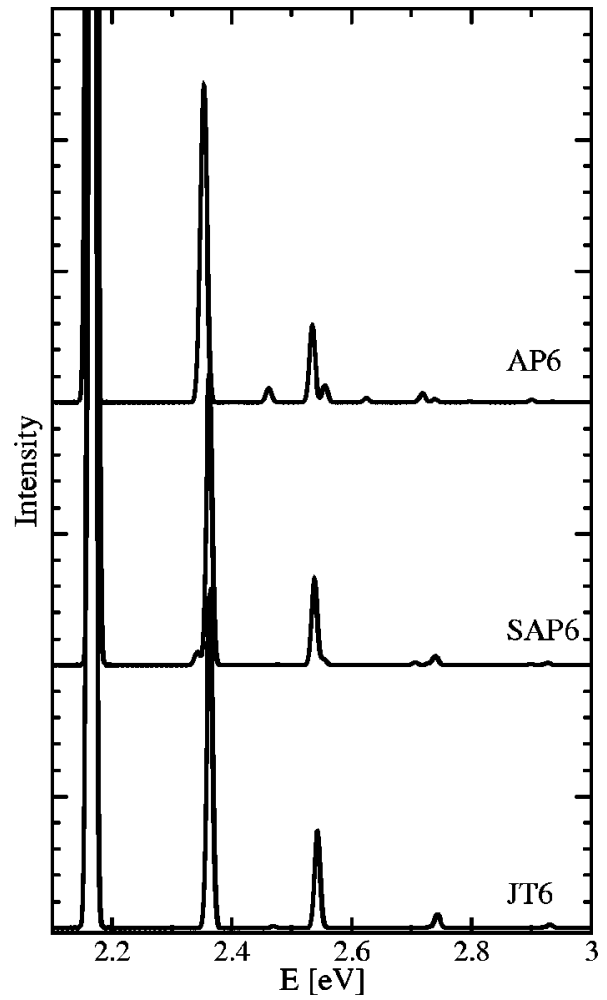

FIG. 9. Absorption spectra obtained after vertical excitation (a damping of $200 \mathrm{fs}$ has been used in the autocorrelation functions). For ease of reading, the spectra have been vertically shifted.

\section{B. Ad hoc approximation (AP6)}

As the least rigorous model an ad hoc approximation has been tested. This approximative model will be referred to as "AP6" in the following. It is obtained similarly to the SAP6 model but to account for the pronounced anharmonicity of the system simple powers of $x$ and $y$ are added on the diagonal instead of the $\mathcal{V}^{(n)}$ matrix elements. The full Hamiltonian potential matrix reads as

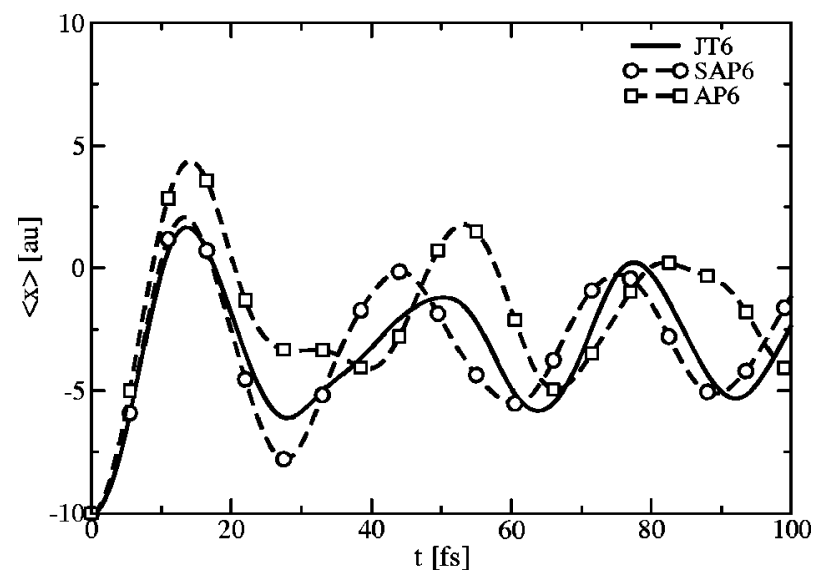

FIG. 10. Evolution of the mean value of $x$ as a function of time in fs for JT6 (full line), SAP6 (dashed line with open circles), and AP6 (dashed line with open squares). 


$$
\begin{aligned}
\mathcal{H}= & \sum_{n=0}^{2} \frac{1}{n !}\left\{\left(\begin{array}{cc}
\mathcal{V}^{(n)} & 0 \\
0 & \mathcal{V}^{(n)}
\end{array}\right)+\left(\begin{array}{cc}
\mathcal{W}^{(n)} & \mathcal{Z}^{(n)} \\
\mathcal{Z}^{(n)} & -\mathcal{W}^{(n)}
\end{array}\right)\right\} \\
& +\sum_{n=3}^{6} \frac{1}{n !}\left(\begin{array}{cc}
\mathcal{S}^{(n)} & 0 \\
0 & \mathcal{T}^{(n)}
\end{array}\right) .
\end{aligned}
$$

The parametrization of the extra diagonal elements of the potential energy cuts along $x$ and $y$ is given by

$$
\begin{aligned}
& \mathcal{S}^{(3)}=s_{x}^{(3)} x^{3}, \\
& \mathcal{S}^{(4)}=s_{x}^{(4)} x^{4}+s_{y}^{(4)} y^{4}, \\
& \mathcal{S}^{(5)}=s_{x}^{(5)} x^{5}, \\
& \mathcal{S}^{(6)}=s_{x}^{(6)} x^{6}+s_{y}^{(6)} y^{6}, \\
& \mathcal{T}^{(3)}=t_{x}^{(3)} x^{3}, \\
& \mathcal{T}^{(4)}=t_{x}^{(4)} x^{4}+t_{y}^{(4)} y^{4}, \\
& \mathcal{T}^{(5)}=t_{x}^{(5)} x^{5}, \\
& \mathcal{T}^{(6)}=t_{x}^{(6)} x^{6}+t_{y}^{(6)} y^{6} .
\end{aligned}
$$

The missing odd powers of $y$ reflect the symmetry with respect to the $\hat{C}_{2}$ rotations and the $\hat{\sigma}_{v}$ mirror planes. The capability of the AP6 model to represent the data points is reflected in the maximum and rms errors which are given in Table V. It is observed that AP6 results in fitting errors slightly larger than JT5 but clearly better than JT4 and SAP6, especially for the absolute errors at higher excess energies. Thus it can be expected that the true adiabatic potentials can be represented well enough by AP6 to yield accurate dynamics results. However, as can be seen from Figs. 8, 9, and 10 the AP6 model shows even larger deviations from the JT6 reference than the SAP6 model. Even worse, it turns out that by deliberately adding terms on the diagonal in the real representation the symmetry of the Hamiltonian is violated. Thus, such ad hoc corrections are to be strictly avoided.

\section{CONCLUSIONS}

In this article the effect of higher order Jahn-Teller coupling terms on the dynamics has been tested. The diabatic Jahn-Teller Hamiltonian matrix (JT6) is presented up to sixth order in both the complex and real representation. Numerical comparative tests have been performed with a twodimensional JT6 Hamiltonian matrix based on the strongly anharmonic $e^{\prime}$ stretching vibration of the ${ }^{2} E^{\prime}$ state of $\mathrm{NO}_{3}$. This JT6 reference model is compared to lower order JahnTeller Hamiltonians and to approximate models which reproduce the anharmonicity along $x$ and $y$ cuts of the potential curves via introduction of ad hoc diagonal terms. The quality of the representation of the JT6 reference by lower order expansions and by the approximative SAPm and AP6 models has been assessed by inspection of the adiabatic potential surfaces and of dynamical quantities. The quality of the representation is found to increase exponentially with the order in the JT expansion. For the presented system and the tested dynamical processes the fourth order model (JT4) is almost converged with respect to the reference whereas the second order approximation is found to be rather inaccurate. The approximative models SAP6 and AP6 correspond to an improvement with respect to the second order model but even if the adiabatic cuts along $x$ and $y$ are found to be nearly identical to the reference curves, the dynamics is significantly different. For both approximations pronounced differences are observed for all dynamical properties, namely in the autocorrelation functions, in the spectra, in the mean position values, and in the electronic adiabatic populations. For the SAP6 model, these differences are related to the unbalanced description in the diabatic potential matrix, i.e., the lack of higher order coupling terms. For AP6, these differences are aggravated due to the fact that AP6 violates the $\hat{C}_{3}$ symmetry requirements. We point out that the cost of creation of the two models JT6 and AP6 is identical since (i) the number of parameters is the same for both models (16 parameters) and (ii) the amount of $a b$ initio energy points in order to determine the parameters is also the same. Thus using the AP6 approximation has no advantage over the use of the correct JT6 Hamiltonian. The creation of the SAP6, needing 14 parameters, is slightly cheaper compared to JT6. However, it leads to a noticeably inferior fitting and the lack of higher order coupling in the diabatic potential matrix has a strong effect on the dynamics.

The dynamical differences observed for the investigated models, particularly the sampling of different configuration spaces and consequently the different evolution of adiabatic populations, are expected to be stronger for larger, realistic systems. For the study of the latter only a very accurate representation of the potential energies and the couplings is likely to yield reasonably accurate results.

The presented examples of AP6 and SAP6 demonstrate that the quality of different diabatization schemes cannot be reliably judged by comparison with a limited set of ab initio data only. To find the deficiencies one would need to sample a more extensive data set on a multidimensional grid. In contrast, the JTn $n=2-6$ models, being based on symmetry considerations and on a systematic and balanced expansion of the potential matrix, lead to much more systematic results. Due to the symmetry restrictions these models are confined to represent data points not even included in the data set and thus fewer data points are needed to obtain reliable representations of the multidimensional potential functions. For these reasons we propose that the use of higher order JTn expansions is preferable over unbalance approaches like SAPm. Application to the physical $\mathrm{NO}_{3}$ system in full dimensionality is in progress. ${ }^{19}$

\section{ACKNOWLEDGMENTS}

The authors thank Professor Wolfgang Domcke for support of this work and for productive discussions. Professor Uwe Manthe is acknowledged for lively discussions and for providing his wave packet propagation code. A.V. is grateful for financial support via a Marie Curie fellowship of the European Community program Improving Human Research Potential and the Socio-Economic Knowledge Base under Contract No. HPMF-CT-2000-00840. W.E. thanks the Leibniz Rechenzentrum for the allocation of large amounts of computer time. 


\section{APPENDIX: PARAMETERS VALUES FOR THE LOWER ORDER JTn MODELS (TABLE VI)}

TABLE VI. Parameters for the "JTn" $n=2-5$ models in (a.u.) obtained with an energy criterion of $4 \mathrm{eV}$ for the fitting data. For the JT2 the values obtained with an energy criterion of $1 \mathrm{eV}$ are given in [ ].

\begin{tabular}{|c|c|c|c|}
\hline$n$ & $a_{1}^{(n)}$ & $\lambda_{1}^{(n)}$ & $\lambda_{2}^{(n)}$ \\
\hline & & JT5 & \\
\hline 0 & $7.411 \times 10^{-02}$ & - & - \\
\hline 1 & - & $2.142 \times 10^{-04}$ & - \\
\hline 2 & $4.015 \times 10^{-05}$ & $-8.660 \times 10^{-06}$ & - \\
\hline 3 & $-2.678 \times 10^{-07}$ & $-3.155 \times 10^{-07}$ & - \\
\hline 4 & $2.518 \times 10^{-08}$ & $-1.416 \times 10^{-09}$ & $4.572 \times 10^{-09}$ \\
\hline \multirow[t]{2}{*}{5} & $-7.325 \times 10^{-10}$ & $-1.110 \times 10^{-11}$ & $2.194 \times 10^{-10}$ \\
\hline & & JT4 & \\
\hline 0 & $7.411 \times 10^{-02}$ & - & - \\
\hline 1 & - & $2.068 \times 10^{-04}$ & - \\
\hline 2 & $4.149 \times 10^{-05}$ & $-9.306 \times 10^{-06}$ & - \\
\hline 3 & $-3.436 \times 10^{-07}$ & $-2.699 \times 10^{-07}$ & - \\
\hline \multirow[t]{2}{*}{4} & $1.986 \times 10^{-08}$ & $-1.529 \times 10^{-09}$ & $7.264 \times 10^{-09}$ \\
\hline & & JT3 & \\
\hline 0 & $7.411 \times 10^{-02}$ & - & - \\
\hline 1 & - & $2.162 \times 10^{-04}$ & - \\
\hline 2 & $4.790 \times 10^{-05}$ & $-6.401 \times 10^{-06}$ & - \\
\hline \multirow[t]{2}{*}{3} & $-2.994 \times 10^{-07}$ & $-2.849 \times 10^{-07}$ & - \\
\hline & & JT2 & \\
\hline 0 & $7.411 \times 10^{-02}$ & - & - \\
\hline 1 & - & $-2.594 \times 10^{-06}\left[1.444 \times 10^{-04}\right]$ & - \\
\hline 2 & $4.326 \times 10^{-05}\left[4.135 \times 10^{-05}\right]$ & $-5.978 \times 10^{-06}\left[-7.483 \times 10^{-06}\right]$ & - \\
\hline
\end{tabular}

${ }^{1}$ C. A. Mead and D. G. Truhlar, J. Chem. Phys. 77, 6090 (1982).

${ }^{2}$ C. A. Mead, J. Chem. Phys. 78, 807 (1983).

${ }^{3}$ T. Pacher, L. S. Cederbaum, and H. Köppel, Adv. Chem. Phys. 84, 293 (1993).

${ }^{4}$ J. Jensen and D. R. Yarkony, J. Chem. Phys. 89, 975 (1988).

${ }^{5}$ D. R. Yarkony, Rev. Mod. Phys. 68, 985 (1996).

${ }^{6} \mathrm{H}$. Werner and W. Meyer, J. Chem. Phys. 74, 5802 (1981).

${ }^{7}$ G. Hirsch, R. J. Buenker, and C. Petrongolo, Mol. Phys. 70, 835 (1990).

${ }^{8}$ W. Domcke and C. Woywod, Chem. Phys. Lett. 216, 362 (1993).

${ }^{9}$ A. J. Dobbyn and P. J. Knowles, Mol. Phys. 91, 1107 (1997).

${ }^{10}$ A. Thiel and H. Köppel, J. Chem. Phys. 110, 9371 (1999).

${ }^{11}$ H. A. Jahn and E. Teller, Proc. R. Soc. London, Ser. A 161, 220 (1937).
${ }^{12}$ H. Köppel, W. Domcke, and L. S. Cederbaum, Adv. Chem. Phys. 57, 59 (1984).

${ }^{13}$ H. C. Longuet-Higgins, Adv. Spectrosc. 2, 429 (1961).

${ }^{14}$ W. Eisfeld and K. Morokuma, J. Chem. Phys. 113, 5587 (2000).

${ }^{15}$ W. Eisfeld and K. Morokuma, J. Chem. Phys. 114, 9430 (2001).

${ }^{16} \mathrm{R}$. Englman, The Jahn-Teller Effect in Molecules and Crystals (WileyInterscience, New York, 1972).

${ }^{17}$ I. B. Bersuker, Vibronic Interactions in Molecules and Crystals (Springer, Berlin, 1989).

${ }^{18}$ R. Janoschek and J. Kalcher, Z. Anorg. Allg. Chem. 628, 2724 (2002).

${ }^{19}$ W. Eisfeld and A. Viel (unpublished). 